\section{Del panamericanismo al ALCA: la difícil senda de las propuestas de una comunidad de intereses en el continente americano (II) ${ }^{1}$}

\author{
From Pan-Americanism to the FTAA: \\ the difficult path of initiatives based on the community \\ of interests in the Americas (II)
}

\author{
José Briceño Ruiz ${ }^{2}$ \\ UNIVERSIDAD DE LOS ANDES \\ MÉRIDA, VENEZUELA \\ $\triangle$ bricenoj@ula.ve
}

\author{
Anuario Latinoamericano \\ Ciencias Políticas \\ y Relaciones Internacionales \\ vol. 4, 2017 \\ pp. $139-162$
}

DOI: 10.17951/al.2017.4.139

\title{
RESUMEN
}

Este trabajo es la segunda parte de un estudio en el cual se analiza las dificultades que han tenido las propuestas de cooperación e integración regional entre Estados Unidos y América Latina, desde la propuesta de una unión aduanera presentada en el I Congreso Internacional Americano (1889-1890) al Área de Libre Comercio de las Américas (ALCA) en la década de 1990. En el artículo se cuestiona el argumento de la existencia de una comunidad de intereses entre los países del continente americano, normalmente utilizado para fundamentar las iniciativas de integración y cooperación en el hemisferio occidental. Realizando un análisis histórico de larga duración, se observa como intereses nacionales, más que la existencia de una comunidad de intereses, han fundamentado las propuestas de cooperación en las Américas. Este trabajo se concentra en el periodo comprendido entre el surgimiento del interamericanismo en los albores de la Segunda Guerra Mundial y el fracaso de la propuesta de crear un Área de Libre Comercio de las Américas (ALCA).

1 Este trabajo es resultado del proyecto de investigación "La persistencia de la integración regional en América Latina: la autonomía política y desarrollo económico como variables explicativas", aprobado y financiado por el Consejo de Desarrollo Científico, Humanístico, Tecnológico y de la Artes (CDCHTA) de la Universidad de los Andes, Mérida, Venezuela. Código E-318-12-09. El autor agradece al CDCHTA por su apoyo a este proyecto.

2 Doctor en Ciencias Políticas del Instituto de Estudios Políticos de Aix-en-Provence, Francia. Profesor Asociado de la Facultad de Ciencias Económicas y Sociales de la Universidad de los Andes, donde es coordinador del Grupo de Estudios sobre Regionalismo, Integración Económica y Desarrollo. 
PALABRAS CLAVE: regionalismo, Estados Unidos, América Latina, interamericanismo, ALCA.

\section{ABSTRACT}

This paper is the second part of a study that analyses the difficulties experienced by the diverse initiatives of regional cooperation and integration between the United States and Latin America - from the proposal of creating a customs union, presented at the First International American Conference (1889-1890), to the Free Trade Area of the Americas (FTAA) in the 1990s. The argument of the existence of a community of interests between the countries of the Americas - normally used to support initiatives of integration and cooperation in the Western Hemisphere - is questioned in this paper. By doing a long-term historical analysis, one observes that national interests, rather than the existence of a community of interests, have inspired the proposals of cooperation in the Americas. This paper focuses on the period between the emergence of inter-Americanism, at the dawn of the Second World War, and the failure of the proposal to create a Free Trade Area of the Americas (FTAA).

KEYWORDS: regionalism, United States, Latin America, inter-Americanism, FTAA.

Como se señaló en la primera parte de este trabajo, publicada en el número anterior de este anuario, en el estudio de las relaciones de Estados Unidos con América Latina han coexistido cuatro posiciones: la idea de una comunidad de intereses, la versión de la irrelevancia creciente, la visión de la voluntad y la práctica imperialista y la idea de la declinación hegemónica. En este trabajo se analiza el periodo que va desde los años previos al estallido a la Segunda Guerra Mundial hasta el colapso del Área de Libre Comercio de las Américas (ALCA). En esta etapa se observan varios sub-periodos en los cuales predominan diversos enfoques sobre la relación bilateral. Entre los años treinta y el fracaso de la Alianza para el Progreso (ALAPRO), fue dominante la idea de una comunidad de intereses en el hemisferio occidental, aunque teñida con fuertes motivaciones estratégicas y con momentos de claro intervencionismo (como plantea la visión de la voluntad y práctica imperialista), ejemplo de lo cual fue el derrocamiento de Gustavo Arbenz en 1954, el apoyo a la invasión de Bahía de Cochinos en 1961 y la invasión de la República Dominicana en 1965.

En la década del setenta, la idea de irrelevancia creciente se vuelve hegemónica, pero ya a fines de la misma y durante los años ochenta, se produce un nuevo periodo de intervencionismo, lo que fue una respuesta a la expansión cubano-soviética en la Cuenca del Caribe. Especialmente durante las dos administraciones de Ronald Reagan, el intervencionismo estadounidense en América Central creció de forma importante, manifestada en el financiamiento a los llamados "Contras", grupo armado que buscaba derrocar al gobierno del Frente Sandinista de Liberación Nacional o el apoyo al gobierno de El Salvador en su conflicto militar con el Frente Farabundo Martí de Liberación Na- 
cional (FMLN). En esa década también se produce la invasión a Grenada en 1983 y Panamá en 1989, aunque ya en ese momento se estaban produciendo cambios en la política hemisférica de Washington.

En efecto, en un contexto global distinto tras el fin de la Guerra Fría y el inicio de un periodo de hegemonía neo-liberal en América Latina, Estados Unidos relanza la idea de una comunidad de intereses en el Hemisferio Occidental, pero en vez de acudir al argumento de enfrentar a un enemigo externo de forma coordinada, esta vez se plantea crear un espacio económico y comercial común en el cual se promovería el desarrollo económico y social, la democracia y la defensa de los derechos humanos. Las Cumbres de las Américas, el relanzamiento de la Organización de Estados Americanos (OEA) y, en particular, la propuesta del ALCA son los elementos centrales de la nueva estrategia estadounidense.

En este trabajo se analiza la evolución de la idea de una comunidad de intereses a lo largo de esos diversos sub-periodos. Para ello, el artículo se divide en cinco secciones. En la primera se analiza el inter-americanismo desde los albores de la II Guerra Mundial a la ALAPRO. En la segunda, se examina el predominio de la visión de irrelevancia después del fracaso de la ALAPRO. En la tercera sección se evalúa la década previa al ALCA, proceso éste que se examina en detalle en la cuarta sección. Las vicisitudes de la integración hemisférica son analizadas en la última sección del trabajo.

\section{El inter-americanismo y el regionalismo americano en la Guerra Fría: De la OEA a la ALAPRO}

La Conferencia de Lima de 1938 -que tiene lugar en el contexto del clima pre-bélico previo a la Segunda Guerra Mundial- significó un giro de la agenda de las conferencias internacionales americanas, pues las cuestiones de la seguridad, defensa y solidaridad continental fueron el centro de las discusiones. Inclusive previo a la Conferencia de Lima, se realizó en Buenos Aires una conferencia extraordinaria de Consolidación de la Paz en 1936, a la que asistió Franklin Delano Roosevelt. En marzo de 1945, cuando las hostilidades no habían cesado aún en Europa y el Pacífico, se realizó la Conferencia Interamericana sobre Guerra y Paz, en Chapultepec, México. Esta preocupación por los temas de seguridad había transformado de manera sustantiva la agenda de las Conferencias, pasando a un segundo plano muchos de los temas presentes desde 1889 y privilegiándose la defensa común frente a enemigos y amenazas externas.

Durante este periodo, la expresión del panamericanismo entró en gradual desuso y se sustituyó por "interamericanismo". Esta expresión describe entonces la etapa de las relaciones entre América Latina y Estados Unidos, en la cual se mantuvo la institucionalidad de las Conferencias, pero el objeto de discusión de éstas fue mayormente sobre temas de seguridad y defensa.
Del panamericanismo al ALCA: la difícil senda de las propuestas de una comunidad de intereses en el continente americano (II)

José Briceño Ruiz 
Una vez concluida la Segunda Guerra Mundial y ya notorias las diferencias entre Estados Unidos y la Unión Soviética con el inicio de la Guerra Fría, se retoman las Conferencias denominadas interamericanas, con la idea de establecer mecanismo frente a lo que Estados Unidos percibía como amenazas para el Hemisferio Occidental (véase Cuadro 1). En este contexto, se realizó en 1947 la Conferencia Interamericana para el Mantenimiento de la Paz y la Seguridad del Continente, realizada en Río de Janeiro, en la cual se suscribió el Tratado Interamericano de Asistencia Recíproca (TIAR). El año siguiente, se realizó en Bogotá la IX Conferencia Internacional Americana, en la cual se estableció la OEA. La creación de la OEA y la firma del TIAR, se trataron fundamentalmente de iniciativas en el ámbito de la seguridad y defensa, sin tener mayores implicancias en términos de comercio y cooperación al desarrollo. Esta agenda de seguridad dominó las cumbres interamericanas hasta la Conferencia realizada en 1954 en Caracas, que cerró el ciclo que se había iniciado en 1889.

Junto con esta especie de "securitización" de la agenda bilateral, este periodo se caracteriza por la denominada política del "buen vecino" aplicada desde el primer gobierno de Franklin Delano Roosevelt (1933-1936), mediante la cual Estados Unidos renunció al intervencionismo que caracterizó sus relaciones con sus vecinos al sur del Río Bravo durante las dos primeras décadas del siglo XX. En este sentido, la "política del buen vecino" era ciertamente una expresión de la idea de una comunidad de intereses en el Hemisferio Occidental, cuyos principios eran la no intervención y la no injerencia en los asuntos internos de los Estados. Sin embargo, como señala Bryce Wood, se trató, en verdad, de la no intervención y la no injerencia de Estados Unidos, que debía abstenerse de tales acciones para que el continente fuese sociedades del siglo $\mathrm{XX}$, iguales desde el punto de vista jurídico y no basadas en el poder militar. “Todos los países contaban con la asistencia económica, los procedimientos de consulta y otras actividades cooperativas que dieran las bases sustantivas para lo que llegaría ser un esfuerzo notable de cooperación internacional en el cual estaban comprometidas las repúblicas americanas" (Bryce 1985: IX).

En los años cincuenta se produjo un significativo retroceso en las relaciones interamericanas. Varios factores explican este retroceso. Un primer elemento fue el fin de la política del buen vecino, en 1954, con la invasión a Guatemala para derrocar al Presidente Arbenz. Además de eso, el énfasis que Estados Unidos daba a la lucha contra el comunismo en su agenda contrastaba con el poco interés en la ayuda al desarrollo económico, un tema de creciente importancia para los gobiernos latinoamericanos. Desde fines de los años cuarenta varios líderes de la región habían solicitado la aplicación de una especie de Plan Marshall para América Latina. La respuesta fue negativa y durante la Conferencia Interamericana de Río de Janeiro de 1947, el Secretario de Estado George Marshall, señaló que no habría ningún Plan Marshall para América Latina, pues el capital disponible para el extranjero debería ser dirigido a Europa Occidental (citado en Bitar 1984: 124). En vez de ello, Estados Unidos 
se limitó a brindar asistencia técnica en campos como la salud, la educación y la agricultura. Ciertamente el Export - Import Bank y el International Bank desarrollaron algunos programas, pero el enfoque estadounidense era que el principal trabajo en favor del desarrollo debía ser realizado por el capital privado, tanto nacional como extranjero. "Para estimular la generación de capital doméstico y flujo de capital extranjero, la tarea principal era mantener o restaurar la estabilidad monetaria y un clima favorable a la inversión. La frase frecuentemente usada era de 'poner orden en casa" (Hirschman 1961: 39).

\begin{tabular}{|c|c|c|}
\hline Fecha & Sede & Temas principales de la agenda \\
\hline 1945 & Chapultepec, México & $\begin{array}{l}\text { Conferencia Interamericana sobre los Problemas de } \\
\text { la Guerra y la Paz }\end{array}$ \\
\hline 1947 & Río de Janeiro, Brasil & $\begin{array}{l}\text { Conferencia Interamericana para el Mantenimiento } \\
\text { de la Paz y la Seguridad del Continente: } \\
\text { Se firma el Tratado Interamericano de Asistencia } \\
\text { Recíproca (TIAR) }\end{array}$ \\
\hline 1948 & Bogotá, Colombia & $\begin{array}{l}\text { IX Conferencia Interamericana: } \\
\text { Se crea la Organización de Estados Americanos; } \\
\text { se firma el Pacto de Bogotá o Tratado Americano } \\
\text { de Soluciones Pacíficas; se aprueba el Convenio } \\
\text { Económico de Bogotá. }\end{array}$ \\
\hline 1954 & Caracas, Venezuela & $\begin{array}{l}\text { Lucha contra el comunismo, asilo diplomático, asilo } \\
\text { territorial y relaciones culturales interamericanas. }\end{array}$ \\
\hline
\end{tabular}

Fuente: elaborado por el autor con base en Luis Dallanegra Pedraza, Relaciones políticas entre Estados Unidos y América Latina, ¿Predominio monroista o unidad americana?, disponible en: http://luisdallanegra.bravehost.com/EUA_Amla/tapausal.htm

Esta política se desarrollaba en el momento en que América Latina vivía un complejo proceso de industrialización y emergencia de gobiernos descritos como nacional-populistas, como el de Juan Domingo Perón en Argentina y Getulio Vargas en Brasil, caracterizados por promover políticas nacionalistas y, especialmente Perón, en muchos aspectos anti-estadounidenses. A esto se sumó luego la revolución cubana,, en 1959, y el posterior alineamiento de Fidel Castro con la Unión Soviética. En este escenario, se produjo un incremento de los movimientos insurgentes en contra de los gobiernos militares en el poder y un creciente aumento del sentimiento anti-estadounidense.

La estrategia de Washington se modificó a partir de 1960, debido a factores como el auge de movimientos insurreccionales de izquierdas inspirados en la triunfante Revolución Cubana. Este cambio en la política estadounidense obedeció a otros factores. En primer lugar, existían motivos estratégicos y de seguridad hemisférica que hacían imperativo favorecer el desarrollo económico,
Del panamericanismo al ALCA: la difícil senda de las propuestas de una comunidad de intereses en el continente americano (II)

José Briceño Ruiz

Cuadro 1.

Conferencias

Interamericanas 1945-1954 
para evitar convulsiones e inestabilidad producto del atraso económico. En segundo lugar, estaban factores de self interest resultado de la percepción que una América Latina unida en un gran mercado ampliado se convertiría en una zona en la que las inversiones y los productos estadounidenses tendrían su mercado. Finalmente, es necesario considerar los problemas de política internacional de la época, en particular el deseo de Washington de crear un modelo que fuese válido para las restantes regiones del mundo en desarrollo, en ese entonces más interesadas en modelos de economías planificadas (Laredo 1968: 72).

En junio de 1958, los presidentes de Colombia, Alberto Lleras Camargo, y de Brasil, Juscelino Kubitschek, enviaron al Presidente Dwight Eisenhower propuestas en las que se establecían un vínculo entre la democracia y el desarrollo, por un lado, y la seguridad hemisférica, por el otro. El presidente Eisenhower respondió enviando una "Fuerza de Trabajo" a Brasil para conversar con los dos líderes latinoamericanos. El resultado fue la "Operación Panamericana”, un programa hemisférico de ayuda para superar el subdesarrollo y la inestabilidad (Tulchin 1988: 10). Esto era el inicio del fin de la estrategia basada en la iniciativa privada y las inversiones, y el comienzo de otro en el cual se escuchaban las demandas latinoamericanas de cooperación económica. En un encuentro de la OEA, realizado en septiembre de 1960, Estados Unidos suscribió por primera vez una convención multilateral en la cual se solicitaba el establecimiento de medidas multilaterales para promover el desarrollo económico y social de los países del continente. Posteriormente, el gobierno estadounidense se comprometió a garantizar fondos -denominado el Fondo Interamericano para el Progreso Social- que servirían para aliviar las condiciones del subdesarrollo. Sin embargo, estos recursos no serían ejecutados sino hasta que el nuevo gobierno de John F. Kennedy (1961-1963) asumiera el poder (Tulchin 1988: 12).

La Alianza para el Progreso (ALAPRO) fue anunciada en la Conferencia del Consejo Interamericano Económico y Social de la OEA, realizada en Punta del Este, Uruguay, en agosto de 1961. Presentada como una alternativa al escenario de un conflicto centro-periferia que formulaba la CEPAL, la ALAPRO se apoyaba en la concepción de la armonía de intereses centro-periferia y en la prédica de "ayudar a ayudarse", lo cual facilitaría el proceso de modernización de los países latinoamericanos y su inclusión en la economía mundial dominada por Estados Unidos (Laredo 1995: 102). Su objetivo era estimular el crecimiento económico de los países latinoamericanos en al menos 2,5\% anual y desarrollar una serie de programas en materia de reforma agraria y fiscal, mejora en los aprovisionamientos de agua, electrificación, programas de alfabetización, vivienda, atención médica, bancos de desarrollo, planes y agencias de planificación, asistencia técnica, reforma en materia educativa, legal, de planificación familiar y militar, y democratización, entre otros. Como señala Joseph Wiarda, se trató de un "programa vasto y ambicioso -demasiado vasto y demasiado ambicioso- como la experiencia lo demostraría. Y fue lanzado con gran fanfarria; la retórica declaraba una revolución democrática y social en América Latina" (Wiarda 1986-1987: 137). 
La ALAPRO fue un gran fracaso. Ya en 1962, su administrador estadounidense, Teodoro Moscoso, afirmaba que "no existía ninguna razón para celebrar el primer aniversario [de la ALAPRO]” (citado en Galeano 1963: 364). Surgieron críticas de diferentes voceros latinoamericanos, como, por ejemplo, la poca asistencia económica efectiva o la insistencia en apoyar proyectos que favorecían a Estados Unidos. Esto condujo al entonces embajador de Brasil en Washington, Roberto de Oliveira Campos, a afirmar en 1963 que: "la ayuda parece estar condicionada a la compra de bienes estadounidenses. Es parte de un programa de expansión de los mercados externos de Estados Unidos, dirigido a absorber el exceso de productos estadounidenses y a controlar la sobreproducción en la industria exportable estadounidense" (citado en Galeano, 1963: 364). Por estas razones, ya a mediados de la década del sesenta, la ALAPRO había perdido su impulso. En 1967, se realizó un último intento por revitalizarla, cuando se convocó una reunión de presidentes americanos en Punta del Este, Uruguay. Posteriormente, se elaboró el denominado Plan de Acción de Viña del Mar, que tampoco produjo mayores resultados (Bitar 1984: 124).

Las causas del fracaso de la ALAPRO fueron diversas. Una de ellas fue la transición de los gobiernos del Presidente Kennedy al de Lyndon Johnson (1963-1969) y de éste a Richard Nixon (1969-1974), durante el cual el interés o el compromiso con la ALAPRO desaparecieron. También las oligarquías y los militares latinoamericanos, poco favorables a medidas como la reforma agraria, fueron culpables del fracaso. De igual manera, los mecanismos internos de aplicación de los proyectos y la poca coordinación entre las instituciones involucradas, tanto en el gobierno de Estados Unidos como en los países latinoamericanos, explican su poco éxito (Wiarda 1986-1987: 136).

\section{Entre la irrelevancia y la condicionalidad: la visión estadounidense después de la ALAPRO}

A fines de los años sesenta y ante el evidente fracaso de la ALAPRO, se inició una nueva etapa en las relaciones bilaterales, en la cual domina el enfoque de la irrelevancia de América Latina en la agenda internacional de Estados Unidos. Esto quedó plasmado en el Informe Rockefeller sobre la Calidad de Vida en las Américas, publicado en 1969, realizado por Nelson Rockefeller, entonces gobernador del Estado de Nueva York, luego de una visita a América Latina. En el informe se establecían ciertos criterios que debían orientar la política estadounidense hacia esta región. En concreto se recomendaba (Bitar 1984: 124):

1. No realizar propuestas que generasen expectativas, señalándose además que la responsabilidad para resolver los problemas económicos era fundamentalmente de países latinoamericanos.

2. Sustituir el paternalismo por el "partnership" o asociación.

3. Concentrarse en el comercio y no en la ayuda, de manera que los productos latinoamericanos tuvieran un mejor acceso en los mercados de Estados
Del panamericanismo al ALCA: la difícil senda de las propuestas de una comunidad de intereses en el continente americano (II)

José Briceño Ruiz 
Unidos. Esto suponía revisar algunas cuotas y disminuir el proteccionismo de Estados Unidos.

4. Canalizar preferentemente la ayuda financiera a través de organismos multilaterales.

5. Estudiar mecanismos para estabilizar los precios de productos primarios.

6. Colaborar en la renegociación de la deuda externa.

7. Estimular la producción manufacturera y el desarrollo tecnológico de América Latina.

Estas propuestas retomaban el enfoque de las relaciones con los países latinoamericanos en el cual la iniciativa privada tenía un papel fundamental y el comercio era un aspecto central. En vez del amplio programa de cooperación multisectorial de la ALAPRO, en la nueva política el énfasis debía estar en la promoción de las exportaciones por parte de los países latinoamericanos y en la creación de una atmosfera favorable a la inversión privada. Si los gobiernos tenían éxitos en alcanzar estos objetivos, Estados Unidos "podría realizar su aporte al desarrollo económico con su mercado y con sus empresas transnacionales" (Bitar 1984: 125).

Este mayor énfasis en las relaciones comerciales fue un elemento fundamental de la política hacia América Latina durante los años sesenta y setenta. Esta fue una época compleja en las relaciones hemisféricas. La crisis interna en Estados Unidos debido a la Guerra de Vietnam, el fin del patrón oro en 1973, el escándalo de Watergate en 1974 y el expansionismo soviético en el Tercer Mundo, hizo que la política hacia América Latina dejase de ser considerada como "una relación especial". Por el lado latinoamericano, la creciente inestabilidad política e insurgencia armada concluyeron con cruentos golpes de Estado en el Cono Sur, mientras que el expansionismo cubano-soviético en Nicaragua, Guyana, Jamaica y Granada convirtió a la Cuenca del Caribe en un terreno de la Guerra Fría, situación que se consolidaría en el primer lustro de la década del ochenta, especialmente en Centroamérica. En este escenario, las condiciones para promover las relaciones comerciales y de cooperación no eran ciertamente las más propicias y en vez de ello se producía un creciente intervencionismo militar estadounidense.

En este periodo se impulsaron algunas iniciativas, aunque sus logros hayan sido finalmente pocos. Una de estas iniciativas fue el establecimiento en 1970 del Comité Especial de Consulta y Negociación (CECON), instancia dirigida a conducir las relaciones económicas entre Estados Unidos y América Latina en el marco de la OEA. Esta instancia constituyó una innovación en las relaciones interamericanas, "pues contemplaba dos partes, América Latina y los Estados Unidos, y no veinte países” (Bitar, 1984: 125). Parecía ser de alguna manera la ruptura de la tradición en la política exterior de Estados Unidos hacia la región que siempre había preferido la vía bilateral con cada uno de los países. Además, en la CECON estaba implícita la idea de "negociación". No obstante, el progreso de la CECON fue limitado, en principio porque sus atribuciones nunca fueron claras y Estados Unidos se mostró en realidad poco 
interesado en una instancia de negociación con América Latina. En vez de ello, Washington mantuvo su tradicional apego al bilateralismo. Pronto la CECON perdió toda importancia y a fines de los setenta había prácticamente desaparecido (Bitar 1984: 125).

En 1974, el Congreso estadounidense aprobó la Ley de Comercio que reguló el Sistema Generalizado de Preferencias (SGP). Existían grandes expectativas en los países latinoamericanos que la SGP permitiría un mayor acceso al mercado de Estados Unidos. Estas expectativas pronto se vieron defraudadas. Por un lado, la lista inicial de productos fue muy limitada. Además, el gobierno estadounidense podía reducir la lista de productos elegibles. Así, en 1977 se limitó la elegibilidad a los países que suministrasen menos de 300 millones de un producto no elegible, y no más de la mitad de las importaciones de ese producto por parte de Estados Unidos. Igualmente, en la Ley Comercial se excluía de los beneficios de la SGP a todos los países de la Organización de Países Exportadores de Petróleo (OPEP) y a los países comunistas. Esto significó la exclusión de Ecuador y Venezuela (Grunwald 1977: 9).

A mediados de los años setenta se elaboró un nuevo estudio sobre las relaciones con América Latina, el denominado Informe Linowitz, elaborado en 1975 por una Comisión presidida por Sol M. Linowitz. Este informe establecería las bases de la política del gobierno de James Carter hacia América Latina. En el informe se reconocía que las relaciones con América Latina debían ser insertadas en un contexto global de creciente interdependencia y crisis económica. De igual manera, se aceptaba que los países de la región habían logrado un importante avance económico que le había permitido incrementar su presencia en el sistema económico internacional. Finalmente, en el Informe se señalaba que Estados Unidos había perdido en términos relativos su poder en la economía mundial (Bitar 1984: 126). En las cláusulas de las relaciones con América Latina, el informe se proponía eliminar las posibles fuentes de conflicto y promover el comercio, lo que, en verdad, no representaba innovar mucho en relación a propuestas previas. El informe sugería varias medidas, entre ellas (Bitar 1984: 126):

1. Rechazar las enmiendas Hickenlooper y González que establecían represalias contra los países que habían nacionalizado activos estadounidenses.

2. Estudiar la posibilidad de establecer un Código de Conducta para las Empresas Transnacionales.

3. Explorar mecanismos para compensar las fluctuaciones de precios y la de demandas de productos básicos.

4. Eliminar el poder de veto de Estados Unidos en el Fondo de Operaciones Especiales del Banco Interamericano de Desarrollo (BID).

5. Apoyar el Sistema Generalizado de Preferencias.

6. Canalizar la ayuda financiera hacia los países más pobres de América Latina y a los grupos de menor ingreso al interior de éstos.

Un elemento crucial en la estrategia propuesta en el Informe Linowitz era contextualizar las relaciones con América Latina en el marco multilateral, su-
Del panamericanismo al ALCA: la difícil senda de las propuestas de una comunidad de intereses en el continente americano (II)

José Briceño Ruiz 
perando la idea de la existencia de una "relación especial" (Bitar 1984: 124). Pocas de las propuestas del Informe Linowitz fueron aplicadas, salvo quizás la desaparición de la idea de una "relación especial". En vez de ello se produjo una declinación de las relaciones económicas bilaterales y la ausencia de iniciativas por parte de Estados Unidos al punto que en 1975 el subsecretario para Asuntos Internacionales del Departamento del Tesoro, Fred Bergsten, señaló que "Estados Unidos no tiene nada que pueda llamarse específicamente una política económica latinoamericana. Más bien, nuestras políticas económicas globales procuran tomar en cuenta íntegralmente las necesidades e intereses de América Latina y consultarlos en su totalidad" (citado en Bitar 1984: 127).

Esto explica el escaso interés de Estados Unidos en promover políticas comerciales y de cooperación económica hacia la región. Las preferencias comerciales fueron limitadas y sólo se concedieron a ciertos países, principalmente los ubicados en la Cuenca del Caribe. En vez de ello, a fines de los años setenta e inicio de los ochenta comienza a desarrollarse una posición más proteccionista que se manifestó en el uso de medidas de salvaguarda y represalias. Igualmente, se extendió la aplicación del concepto de graduación, aplicado cuando las exportaciones de un bien determinado de un país superaban ciertos montos determinados por Estados Unidos o cuando un país imponía condiciones comerciales a las empresas transnacionales estadounidenses (Bitar 1984: 128).

\section{La década previa al ALCA: seguridad y reforma económica}

El ascenso al poder de Ronald Reagan (1980-1988) significó un nuevo periodo en las relaciones de Estados Unidos con América Latina. Para delimitar la estrategia para esta región, se creó la denominada Comisión Santa Fe formada por cinco académicos. En el Informe de Santa Fe (1980), como se conoció el documento final que produciría la Comisión, se destacaba la expansión soviética en la región caribeña. Esta fue la gran preocupación de Reagan, que se inscribía además en una concepción de la política mundial en la cual se planteaba la existencia de un conflicto entre el mundo libre y el bloque comunista. En este conflicto, Estados Unidos, como líder del mundo libre, tenía la obligación de detener la expansión comunista en el Tercer Mundo. Este enfoque se conocería luego como la Doctrina Reagan y, como señala Robert Pastor, "en ninguna parte se aplicó con mayor claridad, coherencia y vigor el punto de vista que Reagan tenía del mundo, que en lo que él denominaba 'patio trasero' norteamericano" (Pastor 1991: 67).

Esto marcó el reinicio de una política de fuerte intervencionismo, especialmente en América Central, expresado en su enfrentamiento al gobierno sandinista y el apoyo al gobierno salvadoreño en contra de la insurgencia del FMLN. Se produjo una creciente intervención militar en América Central y el apoyo que Washington daba a una salida militar a los conflictos en El Salvador 
y Nicaragua era percibido por los países latinoamericanos como un peligro para la estabilidad regional. Se produjo entonces una movilización política de varios Estados que condujo a la creación del Grupo de Contadora ${ }^{3}$ en 1983, pero incluso en América Central surgieron iniciativas propias, especialmente de parte del Presidente de Costa Rica, Oscar Arias, y de Guatemala, Vinicio Cerezo, que serían las que finalmente conducirían al Proceso de Esquipulas (1986-1987) y a la salida negociada del conflicto. En este contexto de contención del comunismo soviético, el gobierno Reagan normalizó sus relaciones con las dictaduras del Cono Sur, con las cuales la administración Carter se había distanciado debido a sus violaciones a los derechos humanos.

Desde el punto de vista de las relaciones económicas y comerciales, las iniciativas del gobierno Reagan hacia América Latina fueron escasas. Apenas se puede mencionar la Iniciativa de la Cuenca del Caribe, aprobada en 1983, y en la cual se concedía ingreso preferencial a ciertos productos de algunos países de América Central y el Caribe insular. Esta iniciativa se inscribía en la política general de contención del comunismo y seguridad nacional. El resto de los países de la región no recibió mayor atención, incluso México, a pesar de que Reagan había manifestado, durante la campaña electoral de 1980, interés en negociar un acuerdo de libre comercio “desde Yukón a Yucatán” (Russell 1994: 193).

La adopción por parte de la administración Reagan de una política económica basada en el juego de las fuerzas del mercado tuvo un significativo impacto en la estrategia internacional estadounidense, que se propuso difundir las "bondades" de un orden económico mundial abierto al comercio y favorable a las inversiones. América Latina, afectada por la crisis de la deuda y la recesión económica, se convirtió en un área en la cual se intentó difundir esta nueva prédica económica. Sin embargo, el discurso favorable a una economía de mercado del gobierno Reagan no encontró mucha recepción en América Latina durante su primera administración. Los países latinoamericanos vivían uno de los periodos más turbulentos de su historia económica después del anuncio del gobierno de México en 1982 de su incapacidad de cumplir con los compromisos de la deuda. A pesar de las exigencias de instituciones como el Fondo Monetario Internacional (FMI) de iniciar reformas estructurales, aún existía una actitud que giraba entre el rechazo y el escepticismo frente a este tipo de políticas. Sería en el segundo lustro de la década del ochenta, cuando la receta neo-liberal comenzaría a imponerse en la región. En el primer lustro, en cambio, la crisis de la deuda fue el tema principal. Y en este asunto predominaba la crítica al gobierno Reagan por su indiferencia ante la crisis. En 1992, el Subsecretario de Estado para Asuntos Interamericanos, Thomas Enders, había señalado que la deuda se trataba "básicamente de una cuestión entre los gobiernos que han solicitado préstamos y los propios mercados" (ci-

3 Deriva su nombre porque su creación fue resultado de una reunión de los Ministros de Relaciones Exteriores de Colombia, México, Panama y Venezuela, realizada en enero de 1983 en la Isla de Contadora, Panamá.
Del panamericanismo al ALCA: la difícil senda de las propuestas de una comunidad de intereses en el continente americano (II)

José Briceño Ruiz 
tado en Pastor 1991: 77). "Sólo cuando la región parecía a punto de establecer un cartel de deudores, en otoño de 1985, el Secretario del Tesoro, James Baker ofreció un plan" (Pastor 1991: 77).

El Plan Baker se proponía ayudar a los países deudores mediante el apoyo financiero tanto de los bancos privados como de la banca de desarrollo, a condición que los países latinoamericanos aplicasen un conjunto de reformas económicas con orientación de mercado. Aunque el Plan Baker fue bienvenido en América Latina, la región solicitaba nuevos créditos y mayores plazos para el pago de la deuda. Con el paso del tiempo, el Plan Baker resultó ser poco efectivo en la resolución de la crisis de la deuda. Por ello, en marzo de 1989, el Secretario del Tesoro del nuevo gobierno de George H. Bush, Nicholas Brady, presentó una nueva propuesta para la reducción de la deuda. Uno de los requisitos de este plan era la adopción de reformas económicas.

Mientras América Latina sufría la tormenta de la crisis de la deuda y la recesión económica, en el gobierno de Estados Unidos se producía un cambio en su estrategia comercial que tendría un profundo impacto en sus relaciones con los países latinoamericanos: la adopción del regionalismo. Estados Unidos optó por diversificar su estrategia comercial y, manteniendo su apoyo a la estrategia multilateral, comenzó a promover acuerdos de integración bilateral y regional, el primero de ellos suscrito en 1985 con Israel. Posteriormente, se continuó la estrategia bilateral al negociar en 1998 un tratado de libre comercio con Canadá (conocido en inglés como CUSFTA). En 1990, el gobierno de George Bush inicia negociaciones con México, a las que se incorporaría luego Canadá, dirigidas a suscribir el Tratado de Libre Comercio de América del Norte (TLCAN). Este fue el inicio de la estrategia regionalista de Estados Unidos en el continente americano, que sería luego ampliada en la propuesta de establecer un Área de Libre Comercio de las América (ALCA). A partir de entonces, la promoción del libre comercio regional se convirtió en el pilar de la estrategia económica de Estados Unidos hacia América Latina, a tal punto que para especialistas como Joseph Tulchin "el Congreso y el público [estadounidense] parecía prestar atención a América Latina principalmente en términos de comercio o relaciones económicas, lo que podría denominarse la 'NAFTAtización' de las relaciones interamericanas” (Tulchin 1997: 34).

\section{El retorno del panamericanismo económico: la integración hemisférica}

En 1988, Ronald Reagan señaló en su Discurso al Congreso sobre el Estado de la Unión: "Nuestra meta será un día cuando el libre flujo de comercio [...] desde la punta de la Tierra del Fuego hasta el Círculo Ártico [...] una a los pueblos del Hemisferio Occidental en un vínculo de intercambio que sea de beneficio mutuo" (citado en Kreklewich 1991: 9). La idea de promover el libre comercio con sus vecinos del sur no era nueva. El 13 de noviembre de 1979, Reagan pro- 
puso un acuerdo norteamericano que producirá "un continente en el cual los bienes y servicios y las personas de los tres países cruzarían las fronteras más libremente" (Ronald Reagan citado en North American Forum on Integration 2008). En enero de 1981, Reagan volvió a sugerir la idea de libre comercio en América del Norte. La exitosa negociación del CUSFTA era un primer paso en la visión de Reagan. Correspondería a George H. Bush continuar la estrategia más allá de América del Norte.

Bush anunció en junio de 1990 la Iniciativa para las Américas (IPA), propuesta que se basaba en tres pilares: el comercio, la inversión y la ayuda en la reducción de la deuda. En el pilar comercial, Bush propuso la creación de una zona de libre comercio desde "Alaska hasta la Tierra del Fuego". En el discurso de la IPA Bush no estableció una fecha definitiva para perfeccionar tal zona de libre comercio, sino que contempló la firma de acuerdos marco con países o grupo de países, en los que se establecerían los pasos para la liberalización comercial. En el pilar de las inversiones, se proponía un programa para incrementar el flujo de las inversiones estadounidenses en América Latina. Para ello se recomendaba la reforma de las legislaciones sobre inversiones extranjeras, lo que sería apoyado mediante un fondo multilateral de 1,5 billones de dólares que se crearía para ayudar a los países que promoviesen la privatización de las empresas públicas. Finalmente, el tercer pilar de la IPA proponía reducir la deuda externa de algunos países latinoamericanos y caribeños. En este sentido, se planteó condonar una parte sustancial de la deuda pública de los países más pequeños de la región, reprogramar a largo plazo el monto restante y permitir su pago en moneda local.

La IPA generó gran optimismo, siendo incluso descrita como el "eje central" en torno al cual se desarrollaría la nueva relación entre el norte y el sur del continente americano (Rojas Aravena 1991: 12). El Presidente argentino Carlos Menem señaló: "estamos viviendo el momento más brillante en nuestras relaciones con Estados Unidos" (citado en Pastor, 1991: 101). El Presidente de Uruguay, Luis Lacalle, llamó a Bush para felicitarlo y expresar el interés de su país en la zona de libre comercio, aseverando "cuando, tras años de quejarnos de que se nos tiene en el olvido, el hombre más importante del mundo nos da la mano [...] entonces, yo pienso que deberíamos tomarla [...] y el brazo, el codo y el hombro también" (citado en Pastor, 1991: 101). El Presidente de Venezuela, Carlos Andrés Pérez, describió a la IPA como "la propuesta más avanzada que Estados Unidos haya jamás presentado a América Latina" (citado en Pastor 1991: 102). Sin embargo, la IPA fue superada por los acontecimientos, en particular el éxito inicial de la negociación del TLCAN. Como señaló Stephen Haggard, en la IPA se planteaba que Estados Unidos firmase acuerdos separados con diferentes grupos regionales, que serían "la piedra fundadora" de una zona de libre comercio hemisférica. Este enfoque no pudo mantenerse una vez aprobado el TLCAN, cuya ampliación se preveía que sería el camino hacia la integración hemisférica. No obstante, esta idea de ampliar el TLCAN también sería superada, adoptándose la estrategia de firmar un
Del panamericanismo al ALCA: la difícil senda de las propuestas de una comunidad de intereses en el continente americano (II)

José Briceño Ruiz 
acuerdo hemisférico global. En una visita realizada en 1993 a México, el VicePresidente Al Gore hizo público el interés de la administración Clinton en convocar una cumbre de Jefes de Estado y de Gobierno para discutir el tema de la integración y cooperación hemisférica. Se iniciaba así una nueva etapa de Cumbres, descrito en inglés por Richard E. Feinberg como summitry in the Americas (Feinberg 1997). Javier Corrales describe este proceso como hemispherism (Corrales 1997: 8), expresión sin traducción textual en español, pero que describía el nuevo ímpetu del regionalismo en el hemisferio occidental.

La agenda de esta fase de integración y cooperación en el continente incluía un proyecto de libre comercio, primera iniciativa de integración comercial de Estados Unidos hacia América Latina desde la Conferencia Internacional Americana de 1889-1890. Además de libre comercio, se discutirían temas como la democracia, la lucha contra la pobreza, la defensa del medio ambiente, la mejora de la calidad de la educación.

A diferencia de la decimonónica propuesta de una unión aduanera, la iniciativa de crear un área de libre comercio de las Américas fue apoyada de forma entusiasta en la mayoría de los países latinoamericanos. Como afirma Javier Corrales, lo que era nuevo era la contrapropuesta de los latinoamericanos, en la cual el libre comercio debería ser no sólo el tema central de la agenda, sino una precondición (Corrales, 1997: 8). Aunque la idea de libre comercio desde Alaska hasta la Patagonia había sido una propuesta de George H. Bush, las dificultades de la ratificación del TLCAN y el complejo fin de la ronda Uruguay del GATT había producido un efecto negativo en la administración Clinton. Se percibía una suerte de fatiga negociadora de acuerdos comerciales, por lo que es válida la afirmación de Corrales en cuanto al impulso que los latinoamericanos dieron a la inclusión del tema comercial en la primera Cumbre de las Américas, denominación oficial del nuevo espacio de encuentro entre Estados Unidos y América Latina. Esta se realizó en diciembre de 1994, en la ciudad de Miami y su resultado fue un ambicioso Plan de Acción en el que se incluían objetivos políticos, económicos y sociales. En materia comercial se propuso crear un Área de Libre Comercio de las Américas, que comenzaría a perfeccionarse a más tardar en el año 2005.

La etapa de negociaciones formales del ALCA se inició en abril de 1998, durante la Segunda Cumbre de las Américas de Santiago de Chile. A partir de la Cumbre Ministerial de Toronto (1989), comenzó formalmente la elaboración de un proyecto de Tratado. Un primer anteproyecto fue presentado en la Tercera Cumbre de las Américas realizada en Quebec, Canadá, en abril de 2001, y un segundo anteproyecto se presentó en la Conferencia Ministerial de Quito, realizada en octubre de 2002.

Sin embargo, luego de la Cumbre de Quebec, se inició un periodo de dificultades en las negociaciones, siendo cada vez mayores y notorias las diferencias entre Estados Unidos y el MERCOSUR, en particular Brasil. La esencia del disenso era el enfoque que existía sobre el ALCA. Estados Unidos apoyaba un modelo OMC plus, según el cual se debía promover el acceso a los 
mercados a través del libre comercio, acompañado por la regulación liberal de temas como los servicios, las compras gubernamentales, la propiedad intelectual, además de la apertura de los mercados agrícolas. Brasil, sus socios del MERCOSUR ${ }^{4}$ y Venezuela después del ascenso de Hugo Chávez al poder, rechazaban tal modelo (véase Briceño Ruiz 2007).

Hubo que esperar a la Cumbre de las Américas de Mar de Plata para que el destino del ALCA fuese decidido. Las diferentes posturas negociadoras que habían dividido al hemisferio y que habían generado la parálisis de las negociaciones desde el 2004 emergieron con toda crudeza en Mar del Plata. Estados Unidos, con el apoyo de 28 países del hemisferio, propuso relanzar las negociaciones del ALCA, lo que fue rechazado por el MERCOSUR con el entusiasta apoyo de Venezuela. La Declaración Final de la Cumbre intentó conciliar estas dos posiciones. Por un lado, se reconoció que algunos miembros sostenían que se debía tener en cuenta las dificultades que ha tenido el proceso de negociaciones del ALCA. A pesar de ello, se debía reconocer la contribución significativa que "los procesos de integración económica y la liberalización del comercio en las Américas pueden y deben aportar al logro de los objetivos de la Cumbre, de crear trabajo para enfrentar la pobreza y fortalecer la gobernabilidad democrática" (Declaración Final de la Cumbre de Mar del Plata 2005). Sin embargo, en la Declaración Final también se reconocía que algunos países sostenían que todavía "no están dadas las condiciones necesarias para lograr un acuerdo de libre comercio equilibrado y equitativo, con acceso efectivo de los mercados, libre de subsidios y prácticas de comercio distorsivas y que tomen en cuenta las necesidades y sensibilidades de todos los socios, así como las diferencias en los niveles de desarrollo y tamaño de las economías" (Declaración Final de la Cumbre de Mar del Plata 2005).

El fracaso de la reunión de Mar del Plata llevó a algunos líderes regionales y analistas a señalar que el ALCA está muerto y que el proyecto estadounidense desapareció. La realidad es algo más compleja pues, aunque ciertamente colapsó el proyecto de suscribir con todos los países del hemisferio un acuerdo comercial "comprehensivo" basado en el modelo TLCAN, Washington modificó su estrategia. En vez de un acuerdo global, optó por promover TLC en los que se establecen las mismas disciplinas que se intentaron regular a través del ALCA.

En cualquier caso, la realidad es que el ALCA ha perdido su relevancia como tema central en la agenda de la integración en el continente americano. El escenario de un "ALCA de dos niveles", que se planteó en Miami (2003), despareció en Mar del Plata. El espacio dejado por el ALCA ha sido ocupado

4 La actitud de los gobiernos de Carlos Menem (1989-1999) y Fernando de la Rua (19992001) respecto al ALCA fue ambigua, aunque terminaron siempre adoptando una posición común con sus socios del MERCOSUR. Con el ascenso al poder de Néstor Kirchner en 2003, el rechazo al acuerdo hemisférico por parte de Argentina fue mucho más claro.
Del panamericanismo al ALCA: la difícil senda de las propuestas de una comunidad de intereses en el continente americano (II)

José Briceño Ruiz 
por los Tratados de Libre Comercio (TLC), que incluso habían comenzado a negociarse desde mucho antes. Ya en 2002 habían concluido las negociaciones con Chile. Posteriormente se iniciaron discusiones para la firma de un TLC con los países de América Central y República Dominicana, conocido como CAFTA, que concluyeron en 2004. Después, en noviembre de 2003, se anunció el inicio de negociaciones de un TLC con Colombia, Perú, Ecuador y Bolivia, aunque estos dos últimos países se abstuvieron luego de participar. Perú concluyó las negociaciones a fines de 2006 y el acuerdo fue ratificado en 2007. Las negociaciones del acuerdo con Colombia concluyeron en 2006, pero el acuerdo fue ratificado apenas en 2011 por el Congreso de Estados Unidos.

La Cumbre de las Américas de Trinidad y Tobago (2009) generó grandes expectativas debido a la presencia de Barack Obama, primer presidente afroestadounidense. La participación de Obama ocurría en un contexto regional completamente distinto al existente cuando se inició la etapa de Cumbres de las Américas en 1994, pues había desaparecido el consenso favorable a las políticas de mercado y, en consecuencia, la idea de integración hemisférica había perdido fuerza. A esto se sumaba la compleja forma como la administración Bush trató la emergencia de líderes como Hugo Chávez en Venezuela, Evo Morales en Bolivia, Rafael Correa en Ecuador. En el ámbito de la integración y cooperación regional esto se ha manifestado en iniciativas como la Unión de Naciones Sudamericanas (UNASUR), la Alianza Bolivariana para los Pueblos de América (ALBA) y la Comunidad de Estados Latinoamericanos (CELAC), que se proponen crear espacios exclusivamente latinoamericanos para incrementar la interdependencia económica y la concertación política.

Todos estos factores marcaron la Cumbre de las Américas de Trinidad y Tobago, en la cual la idea de un bloque regional prácticamente no fue objeto de discusión y fue sustituida por una agenda más pragmática que se desarrollaría con aquellos países interesados. Como señala Abraham Lowenthal (2010: 35), "en lugar de retomar la retórica altisonante de colaboración desde Alaska a Tierra de Fuego, la nueva administración prefería trabajar con los gobiernos de América Latina y el Caribe en algunos temas que se pudieran abordar rápidamente...”.

El tema comercial desapareció casi completamente de la Cumbre de las Américas, realizada en Cartagena de Indias, Colombia, en la cual las discusiones se centraron más en temas políticos como la exclusión de Cuba de estos encuentros, o el reclamo de Argentina a la soberanía de las Islas Malvinas. Esto ocurrió en el marco de un creciente desinterés de Estados Unidos por la región durante la administración Obama, que puede describirse como un nuevo ciclo de irrelevancia de la región en la estrategia global de ese país (véase Whitehead y Nolte 2012). La posible excepción a esto fue la normalización de las relaciones con Cuba en diciembre de 2014 y su ingreso a la Cumbre de las Américas realizada en Panamá en 2015. 


\section{Explicando las vicisitudes del "regionalismo hemisférico"}

Iniciativas como la ALAPRO o el ALCA no tuvieron mejor destino que las propuestas panamericanas de fines del siglo XIX y las primeras décadas del siglo XX. La política del buen vecino fue abandonada en los años 50 y la lógica de la Guerra Fría consolidó la condición de América Latina como una zona de influencia estadounidense en la cual se debía contener cualquier forma de expansionismo comunista. La ALAPRO no fue una iniciativa de integración, sino un mecanismo de cooperación enfocado en los temas sociales que, como señala el especialista francés Alain Rouquié, “marcó época más por su retórica que por sus resultados" (Rouquié 2009: 390). En realidad, esta propuesta tuvo cierto apoyo interno en sectores políticos en Washington, pero la fuente de este apoyo no era la existencia de una comunidad hemisférica de intereses, sino la lucha contra el comunismo y los esfuerzos para limitar que se replicase el modelo cubano en otros países de América Latina y el Caribe. La ALAPRO era el costo que Estados Unidos debía pagar para asegurar la libertad en la región y mantenerla fuera de la influencia soviética. Abraham Lowenthal plantea el asunto en los siguientes términos: "la Alianza para el Progreso fue emprendida en 1961 por un régimen que proclamaba que los norteamericanos estaban dispuestos a 'pagar cualquier precio, sobrellevar cualquier carga [...] para asegurar la supervivencia y el buen éxito de la libertad.' Para 1970, los norteamericanos evidentemente se habían cansado de los costos y las cargas de los compromisos contraídos con el extranjero y se habían vuelto escépticos en cuanto a sus beneficios" (Lowenthal 1970: 395). Posteriormente, Lowenthal señala que "a pesar de 'la idea de Hemisferio Occidental' que dice que América del Norte y del Sur están enlazados por una relación especial y estrecha, por un pasado y aspiraciones en común, la experiencia de esta década ha subrayado el hecho que existen profundas diferencias en cultura, actitudes, fines e intereses entre las naciones de América Latina, y entre éstas y Estados Unidos" (Lowenthal 1970: 398).

Incluso en las discusiones en el Congreso sobre la ALAPRO se evidenciaron las diferencias entre las dos regiones del continente americano en cuanto al tema de la estrategia de desarrollo económico, pues mientras en Estados Unidos era hegemónico el discurso que el crecimiento debía ser impulsado por la actividad privada y las fuerzas del mercado, en América Latina, en esos años fuertemente influenciada por las ideas cepalistas, se otorgaba un papel destacado a la planificación estatal. La ALAPRO admitía ciertas formas de intervención pública, un factor que causó mucha controversia, al punto que el Congreso estadounidense debatió por tres meses si tal planificación era democrática o no (Tulchin 1988).

Finalmente, en las negociaciones del ALCA tampoco existió un apoyo generalizado a esta iniciativa comercial en la sociedad y el mundo político estadounidense. Richard Feinberg, por ejemplo, ha argumentado que fueron
Del panamericanismo al ALCA: la difícil senda de las propuestas de una comunidad de intereses en el continente americano (II)

José Briceño Ruiz 
los países latinoamericanos quienes presionaron a la administración Clinton para que acordase iniciar negociaciones dirigidas a crear una zona de libre comercio. Según Feinberg, Clinton aceptó las demandas latinoamericanas cuando se entendió que la credibilidad de la Cumbre que luego se realizaría en Miami en 1994 sería afectada si no se anunciaba la fecha para iniciar la negociación comercial (véase Feinberg, 1997; 2003). Más que una visión de una comunidad de intereses, fueron los intereses nacionales los que condujeron a Washington a impulsar la integración económica con sus vecinos del Sur. La creciente competencia económica global luego del fin de la Guerra Fría, el escenario de un mundo dividido en bloques comerciales y la necesidad de asegurar un marco legal para sus empresas transnacionales fueron las razones básicas que llevaron a los gobiernos estadounidenses a impulsar el ALCA. Cuando en el proceso de negociaciones se observó que muchas de las demandas de ciertos países, como los miembros del MERCOSUR, podían hacer muy costoso el apoyar al ALCA, el interés por esta propuesta disminuyó en Estados Unidos. Esto explica por qué este país fue tan reacio a realizar concesiones durante la negociación hemisférica. No se observa entonces una comunidad de intereses, sino realpoltik en la construcción del ALCA. El mito de la comunidad de intereses fue negado por el solo hecho que, al mismo tiempo que negociaba el ALCA, Estados Unidos impulsaba la creación de otro bloque regional en otra de sus zonas de influencia: el Foro de Cooperación Asia-Pacifico (APEC) y por recientes iniciativas de negociar mega-bloque como el Acuerdo de Asociación Transpacífico (TTP) o el Acuerdo Transatlántico de Comercio en Inversiones (TTIP). Como en el caso del ALCA, la promoción de estos acuerdos responde a una simple lógica política y estratégica y no a una idea de comunidad de intereses.

Esta breve síntesis nos permite demostrar que en diversos momentos del "regionalismo hemisférico" (la política del buen vecino, la ALAPRO y el ALCA), la idea de una comunidad de intereses pudo alimentar el discurso de la época, pero no fue suficiente para asegurar el éxito de las iniciativas propuestas. En ellas, entraron en juego variables de poder o la promoción de los intereses económicos de los actores políticos y económicos estadounidenses. Cuando existía una convergencia de intereses, esos procesos tuvieron un éxito relativo. Así ocurrió en 1961, cuando John F. Kennedy supo "vender" a la ALAPRO, o en el primer lustro de 1990, cuando George Bush y Bill Clinton aprovecharon el escenario mundial y regional favorable a la apertura para impulsar la integración económica hemisférica. Cuando esa convergencia desapareció, las propuestas de unidad económica regional se desvanecieron o debilitaron, y los argumentos de una comunidad de intereses fueron irrelevantes.

En este sentido, acudiendo a la clasificación de Russell, la mirada de las élites estadounidenses se ha basado más bien en una idea imperialista o en la irrelevancia de la región, que en un enfoque de una comunidad de interés. Este último ha aparecido en momentos bastante delimitados de la relación bilateral y sus logros han sido muy limitados. 
En el caso de América Latina, la resistencia frente a las propuestas estadounidenses ha obedecido a razones distintas. Dos variables han estado históricamente vinculadas al impulso de propuestas de integración económica y de cooperación política: el desarrollo y la autonomía (véase Briceño Ruiz 2012). El concepto e idea de desarrollo económico y su vinculación con la integración data de las mismas propuestas decimonónicas, pero la formulación de una doctrina latinoamericana sobre el desarrollo económico y su vinculación con la integración regional comienza a producirse en el siglo XX. Corresponde a Raúl Prebisch y la Comisión Económica para América Latina realizar los primeros aportes a fines de los cuarenta y durante los años cincuenta del siglo XX. La división del sistema económico internacional en centro-periferia, la articulación de la creación de un espacio regional a través de la integración como mecanismo para promover la transformación productiva (es decir: la industrialización), la preocupación permanente por la distribución de los frutos del progreso técnico y la consideración a los problemas estructurales que impedían el desarrollo económico, son temas objeto de reflexión de quienes proponen una integración regional al servicio de la promoción del desarrollo económico (Prebisch 1959; 1963).

La idea de autonomía política también es de vieja data. La convocatoria del Congreso de Panamá en 1926 por Simón Bolívar se proponía crear una institucionalidad regional (una Confederación de Estados) que asegurase a los entonces nacientes Estados garantizar su existencia frente a cualquier intento de reconquista por la Santa Alianza, además de permitir su viabilidad nacional. Esto no es otra cosa que complementar la independencia adquirida con una verdadera autonomía. Ahora bien, al igual que con la idea de desarrollo económico, la conceptualización de la autonomía se comenzó a realizar también en el siglo XX. Las reflexiones de Víctor Raúl Haya de la Torre o Manuel Ugarte se pueden considerar como referencias, pero la discusión de la cuestión de la "autonomía" se realiza a partir de la década de los cincuenta en tres ejes distintos: el pensamiento nacionalista del Instituto Superior de Estudios Brasileiros (ISEB), cuya figura más conocida es Helio Jaguaribe; la denominada Escuela de la Dependencia y el pensamiento de Juan Carlos Puig en Argentina y los trabajos de Jaguaribe desde fines de la década del sesenta. Aunque en la Escuela de la Dependencia existió una suerte de desdeño hacia la idea de integración latinoamericana, ésta sí fue parte de la reflexión de Jaguaribe y de Puig, que la asociaron a la búsqueda de la autonomía, es decir, la ampliación de los márgenes de maniobra de los países de la región en el sistema internacional (véase Briceño Ruiz 2012).

En sectores políticos, intelectuales y académicos en América Latina existe un problema con las iniciativas de integración hemisférica: no son percibidas como funcionales para lograr los objetivos históricos arriba mencionados, es decir, el desarrollo económico y social y la autonomía política.

Existe en América Latina la percepción que las instancias propuestas por Estados Unidos, como la OEA y la ALAPRO ayudan muy poco para ampliar
Del panamericanismo al ALCA: la difícil senda de las propuestas de una comunidad de intereses en el continente americano (II)

José Briceño Ruiz 
el margen de maniobra de la acción internacional de los países latinoamericanos. Igual rechazo provocó en sectores autonomistas latinoamericanos la ALAPRO. Se debe recordar que la "ALAPRO sólo puede entenderse en el contexto de la Guerra Fría en América Latina y por la necesidad de Estados Unidos de abortar la potencial expansión de la influencia cubana. Inspirada en las tesis de Rostow del crecimiento económico por etapas, planteaba que superando el atraso económico se lograría evitar el descontento social, caldo de cultivo para el desarrollo de los movimientos comunistas" (Morgenfeld 2012: 142). La respuesta latinoamericana fue aceptar la propuesta, si ella promovía la reforma agraria, la mejora de los índices de desarrollo social y el cambio político democrático, pero no debía subordinarse a una mayor dependencia con relación a la economía de Estados Unidos. Esos objetivos no se lograron $y$, en vez de ello, se radicalizaron movimientos subversivos que se enfrentaron a los gobiernos existentes y a la hegemonía estadounidense, lo que abrió el camino a las dictaduras represivas de la década del setenta. La mayor parte de éstas estuvieron en buena medida subordinadas a poderes externos y fueron contrarias a cualquier discurso autonomista.

El caso del ALCA, mucho más reciente, se produce en un contexto de mayor movilización de las sociedades latinoamericanas, organizadas incluso en redes como la Alianza Social Continental. En su fase final, las negociaciones del ALCA también coincidieron con la emergencia de una nueva oleada nacionalista expresada en el ascenso al poder de gobiernos como los de Hugo Chávez, Luis Ignacio Lula da Silva y Néstor Kirchner. En la visión de estos actores, el ALCA no era un mecanismo para autonomizar a la región. Incluso, antes que ellos, la recepción del ALCA por parte de Brasil fue poco entusiasta, como lo evidenció Fernando Henrique Cardoso en la Cumbre de Quebec al señalar que el "El ALCA es una opción, el MERCOSUR un destino." Como señala Tullo Vigevani y sus colaboradores, esta postura no era una "adhesión no crítica, ni siquiera una adhesión, sino una posibilidad deseable de mejorar la posición relativa del país. Se trata de una exigencia que surgió en un país que busca una presencia internacional relevante y capaz de incidir en el orden mundial y, sobretodo, de influir en las reglas y en las decisiones que pesaran sobre su destino, en un medio asimétrico e inhóspito" (Vigevani y Fernandes de Oliveira 2003: 51-52). Los países del MERCOSUR, Venezuela y amplios sectores de las sociedades latinoamericanas y caribeñas compartían este enfoque.

En varios sectores de las sociedades latinoamericanas predominaba la percepción que existía una asimetría o desequilibrio en la negociación del ALCA y se le percibía como una manifestación de los intereses de Estados Unidos que tendría impactos negativos en diferentes sectores sociales, la industria, en regiones atrasadas e incluso en la capacidad de los Estados de regular el funcionamiento de su propia economía interna (Estay, 2005: 71). En otras palabras, en vez de un mecanismo para ampliar la autonomía, el ALCA era visto como fuente de una mayor subordinación frente a Estados Unidos. 
Tampoco se percibía que el ALCA sirviese al desarrollo económico de América Latina, el otro objetivo históricamente vinculado a la integración en esta región. En círculos políticos y económicos se percibía que los ganadores del proceso del ALCA serían "el capital multinacional y pequeños sectores capitalistas vinculados a éste” (Sánchez Daza 2007: 273). La opinión de Norman Girvan puede describir bastante bien la visión del ALCA predominante en esos años. Para Girvan la integración debe: "I) abrir oportunidades para un desarrollo acelerado de los países y regiones menos desarrolladas (incluyendo las regiones dentro de los países); II) abordar las vulnerabilidades de los países más pequeños, las cuales se derivan de sus dimensiones reducidas y III) incluir mecanismos de compensación para los "perdedores" como consecuencia de la integración del mercado, como son el seguro social, programas de reentrenamiento del trabajo y esquemas de crédito para la reconversión industrial" (Girvan 2003: 1). Para Girvan, en el ALCA no se promovía políticas para alcanzar esos objetivos. Por ello, Girvan consideraba necesario pensar "en patrones alternativos para la integración de América Latina y el Caribe que estén orientados específicamente a elevar los niveles de desarrollo y las capacidades competitivas de las economías más pequeñas y menos desarrolladas, al tiempo que se aborda la agenda social" (Girvan 2003: 1). Helio Jaguaribe, por su parte, consideraba que el desequilibrio implícito en el ALCA llevaría a los países de la región a lo que ellos eran hasta la década del treinta del siglo XX: "meros exportadores de productos primarios, con una enorme declinación del PIB y los niveles de empleo". En síntesis, para estos especialistas y para una buena parte de las sociedades latinoamericanas, el ALCA no era un mecanismo para promover el desarrollo económico.

\section{Conclusiones}

El análisis histórico realizado en este trabajo evidencia las dificultades que han sufrido las iniciativas de integración y cooperación de Estados Unidos hacia América Latina. Algunos patrones de acción pueden delimitarse y son los siguientes: desinterés de la sociedad estadounidense en esas propuestas, desconfianza o, en el mejor de los casos, escepticismo en los países latinoamericanos frente a su vecino del norte. Estos factores han tenido un peso mayor en el destino de las iniciativas de "integración y cooperación hemisférica" que el discurso sobre la existencia de una real o supuesta comunidad de intereses.

La experiencia histórica señala que en Estados Unidos la política hacia América Latina ha estado más bien influenciada por la promoción de sus intereses nacionales y por factores ligados a la configuración de poder en el sistema internacional. El surgimiento del panamericanismo en 1889-1890 fue expresión del creciente poder económico de Estados Unidos, cuyas élites ya percibían que podían retar la hegemonía británica en el continente americano y establecer un espacio aduanero común bajo su liderazgo. La creación de la
Del panamericanismo al ALCA: la difícil senda de las propuestas de una comunidad de intereses en el continente americano (II)

José Briceño Ruiz 
OEA o el impulso de la ALAPRO fueron parte de una estrategia global de contención de comunismo durante el periodo de la Guerra Fría. El ALCA fue una respuesta a un escenario global cambiante en el cual los temas económicos parecían dirigir la agenda internacional, pero que se transformó luego de los ataques terroristas del 11 de septiembre de 2001.

Estas propuestas encontraron una buena recepción inicial en América Latina porque existía cierto grado de apoyo en su clase política. Así, tanto la OEA como la ALAPRO fueron apoyadas por gobiernos latinoamericanos que temían que su estabilidad política fuese perturbada por movimientos insurreccionales de izquierda apoyados por la Unión Soviética. Igualmente, gobiernos neo-liberales en los años noventa mostraron un enorme entusiasmo con el ALCA y el modelo de políticas económicas impulsadas por Washington. No obstante, la pobre evolución de iniciativas como la ALAPRO o la asimetría implícita en iniciativas como el ALCA pronto terminaron por desilusionar a los latinoamericanos, lo que causó una pérdida de apoyo.

En el fondo, para los países de América Latina cualquier iniciativa de integración y cooperación "hemisférica" será apoyada, de forma consistente y duradera, sólo si contribuye a su desarrollo económico y a incrementar su voz en los asuntos políticos mundiales. Hacer converger esto con la concepción que tiene Estados Unidos de su papel en la región y en el mundo no ha sido fácil y existen razones para pensar que no lo será en el futuro inmediato. En consecuencia, más allá de la retórica de una comunidad de intereses, la realpolitik ha puesto límites a las relaciones entre Estados Unidos y América Latina. Mientras la actual estructura de poder y las ideas sobre la conveniencia de su mutua cooperación no se transformen, existen razones para pensar que tal situación no se modificará.

\section{Bibliografía}

Baldwin R. E. (1993), Change in the Global Trading System. A Response to Shifts in National Economic Power, en: Protectionism and World Welfare, S. Dominick (ed.), Cambridge University Press, Cambridge.

Bitar S. (1984), De la Alianza para el Progreso a la magia del mercado. Política económica de los Estados Unidos hacia América Latina, "Desarrollo Económico", vol. 24, no 93, abriljunio, pp. 123-137.

Briceño Ruiz J. (2007), La integración regional en América Latina y el Caribe. Procesos históricos y realidades comparadas, Universidad de los Andes, Mérida.

Briceño Ruiz J. (2012), Autonomía y desarrollo en el pensamiento integracionista latinoamericano, en: Integración Latinoamericana y Caribeña. Política y Economía, J. Briceño Ruiz, A. Rivarola Puntigliano, Á. M. Casas Gragea (eds.), Fondo de Cultura Económica, Madrid.

Corrales J. (1997), Why Did It Take 200 Years? The Intellectual Journey to the Summit of the Americas, en: Summitry in the Americas. A Progress Report, R. E. Feinberg (coord.), Institute for International Economics, Washington. 
Dallanegra Pedraza L. (2008), Relaciones Políticas entre Estados Unidos y América Latina: ¿Predominio Monroista o Unidad Americana?, 21-11-2008, http://luisdallanegra.bravehost. com/EUA_Am-la/capit_4.htm, fecha de consulta: 21.10.2008.

Estay J. (2005), A ALCA e suas transformações, en: Globalização e Integração nas Américas. Hegemonia e Contra-Hegemonia, T. Dos Santos (coord.), Edições Loyola, vol. 4, São Paulo.

Feinberg R. E. (1997), Summitry in the Americas. A Progress Report, Institute for International Economics, Washington.

Feinberg R. E. (2003), The Political Economy of the United States' Free Trade Agreements, "World Economy", vol. 26, pp. 1019-1040.

Galeano E. (1963), The Alliance for Progress: Notes for an Obituary, "Monthly Review", noviembre, pp. 364-370.

Girvan N. (2013), Los vacíos del ALCA, http://www.normangirvan.info/wp-content/ uploads/2007/11/vacios-del-alca.pdf, p. 1, fecha de consulta: 17.02.2016.

Grunwald J. (1977), Reflexiones acerca de América Latina en la economía mundial, "Integración Latinoamericana", octubre, pp. 3-14.

Hirschman A. O. (1961), Latin American Issues: Essays and Comments, Twentieth Century Fund, New York.

Jaguaribe H. (2003), General Introduction, en: The European Union, MERCOSUL and the New World Order, H. Jaguaribe, A. Vasconcelos (eds.), Frank Cass, London.

Kreklewich R. J. (1991), North American Integration: Interplay of World Order, State \& Production, "Occasional Papers in Latin American and Caribbean Studies", no 2, abril, Centre for Research on Latin America and the Caribbean (CERLAC), York University, Ontario.

Krugman P. (1995), Regionalism versus Multilateralism: Analytical Notes, en: New Dimensions in Regional Integration, J. De Melo, A. Panagariya (eds.), Centre for Economic Policy Research, Cambridge.

Laredo I. M. (1968), Posición de los Estados Unidos frente a la integración de América Latina: afirmaciones y tendencias, "Revista de la Integración", no 3, noviembre, pp. 58-104.

Laredo I. M. (1995), Trasfondo político de la integración económica, "Aportes para la Integración Latinoamericana", vol. 1, no 1, julio, pp. 99-112.

Lowenthal A. (1970), La Alianza para el Progreso en retrospectiva. Hacia mejores políticas norteamericanas para la década de 1970, "Foro Internacional", México, abril-junio, pp. 392-406.

Lowenthal A. F. (2010), El gobierno de Obama y las Américas: promesa, desengaño, oportunidad, en: Obama y las Américas ¿Esperanza o decepción?, A. F. Lowenthal, L. Whitehead, T. J. Piccone (eds.), Planeta, Bogotá.

Morgenfeld L. (2012). Desarrollismo, Alianza para el Progreso y Revolución Cubana. Frondizi, Kennedy y el Che en Punta del Este (1961-1962), "Ciclos en la Historia, la Economía y la Sociedad”, vol. 20, no. 40, julio-diciembre, pp. 133-165.

North American Forum on Integration, http://www.finanafi.org/eng/integ/chronologie. asp?lan-gue=eng\&menu=integ, fecha de consulta: 24.10 .2008 .

Pastor R. (1991), El Remolino. Política exterior de Estados Unidos hacia América Latina y el Caribe, Siglo XXI Editores, México.

Prebisch R. (1959), El Mercado Común Latinoamericano, "Comercio Exterior", vol. IX, septiembre, pp. 509-513.
Del panamericanismo al ALCA: la difícil senda de las propuestas de una comunidad de intereses en el continente americano (II)

José Briceño Ruiz 
Prebisch R. (1963), Hacia una nueva dinámica del desarrollo latinoamericano, Fondo de Cultura Económica, México.

Puig J. C. (1980), Doctrinas internacionales y autonomía latinoamericana, IEAL-USB, Caracas.

Rojas Aravena F. (1991), ¿Hacia una nueva doctrina en la relación Estados Unidos-América Latina? La Iniciativa para las Américas, "Cono Sur", vol. 10, no 4, julio-agosto, pp. 12-17.

Rouquié A. (2009), América Latina. Introducción al Extremo Occidente, Siglo XXI Editores, México.

Russell J. W. (1994), Nuevas percepciones sobre México en Estados Unidos (1981-1992), en: La nueva relación de México con América del Norte, E. Morales Aragón, M. C. Dávila Pérez (coords.), UNAM, México.

Sánchez Daza G. (2007), El Acuerdo para establecer el Área de Libre Comercio de las Américas: ¿Una integración subordinada a los intereses estadounidenses?, en: Globalización y bloques económicos: mitos y realidades, J. L. Calva (coord.), Miguel Ángel Porrúa-UNAM, vol. 1., México.

Tulchin J. (1997), Hemispheric Relations in the 21st Century, "Journal of Interamerican Studies and World Affairs", vol. 39, no 1, primavera, pp. 33-43.

Tulchin J. S. (1988), The United States and Latin America in the 1960s, "Journal of Interamerican Studies and World Affairs", vol. 30, no 1, primavera, pp. 1-36.

Vigevani T., de Oliveira M. F., Cintra R. (2003), Política externa no periodo FHC: a busca de autonomia pela integração, "Tempo Social", vol.15, no 2, pp. 31-61.

Whitehead L., Nolte D. (2012), The Obama Administration and Latin America: A Disappointing First Term, "GIGA Focus", German Institute for Global and Area Studies, no 6, Hamburgo.

Wiarda H. J. (1986-1987), Misreading Latin America: Again, "Foreign Policy", no 65, invierno, pp. 135-153.

Wood B. (1985), The Dismantling of the Good Neighbor Policy, University of Texas Press, Austin. 